\title{
On sign-changeable interaction in FLRW cosmology
}

\author{
Fabiola Arevalo $^{1, \mathrm{a}}$, Antonella Cid ${ }^{2, \mathrm{~b}}$, Luis P. Chimento ${ }^{3, \mathrm{c}}$, Patricio Mella ${ }^{4, \mathrm{~d}}$ \\ ${ }_{1}^{1}$ Núcleo de Matemática, Física y Estadística, Facultad de Estudios Interdisciplinarios, Universidad Mayor, Casilla 4801043, Temuco, Chile \\ ${ }^{2}$ Grupo Cosmología y Partículas Elementales, Departamento de Física, Universidad del Bío-Bío, Casilla 5-C, Concepción, Chile \\ ${ }^{3}$ Departamento de Física, Facultad de Ciencias Exactas y Naturales, Universidad de Buenos Aires e IFIBA, CONICET, Ciudad Universitaria, \\ Pabellón I, 1428 Buenos Aires, Argentina \\ ${ }^{4}$ Instituto de Ciencias Físicas y Matemáticas, Universidad Austral de Chile, Casilla 567, Valdivia, Chile
}

Received: 24 January 2019 / Accepted: 11 April 2019 / Published online: 24 April 2019

(C) The Author(s) 2019

\begin{abstract}
We investigate an interacting two-fluid model in a spatially flat Friedmann-Lemaître-Robertson-Walker (FLRW) Universe, when the energy transfer between these two dark components is produced by a factorizable nonlinear sign-changeable interaction depending linearly on the energy density and quadratically on the deceleration parameter. We solve the source equation and obtain the effective energy densities of the dark sector and their components. We show that the effective equation of state of the dark sector includes some of the several kinds of Chaplygin gas equations of state as well as a generalization of the polytropic equation of state. We use Bayesian statistics methods to constrain free parameters in the models during the most recent evolution considering supernovae type Ia and measurements of the Hubble expansion rate. The resulting constraints provide new information on sign-changeable interactions, its equivalences and compatibility with previous models and novel late time universe dynamics.
\end{abstract}

\section{Introduction}

Modern cosmological observations indicate that the Universe appears to be in a period of accelerated expansion, first noted in Ref. $[1,2]$ and confirmed by latest observations [3-5]. A plethora of theoretical models have been proposed, in which the present acceleration has been modeled by the so-called dark energy component with a characteristic negative pressure that induces late time acceleration in FriedmanLemaître-Robertson-Walker models [6]. In addition, it was shown that the cosmological interaction between dark matter

\footnotetext{
a e-mail: fabiola.arevalo@umayor.cl

be-mail: acidm@ubiobio.cl

ce-mail: chimento@df.uba.ar

de-mail: patricio.mella@uach.cl
}

and dark energy could address the late time acceleration of the universe and alleviate the coincidence problem presented in the standard cosmological scenario [7-9]. In the literature there are several studies on cosmological interacting models [10-20]; for a general review summarizing this work, see [21]. An interesting aspect of some interacting scenarios consists of alleviating the recently observed tension between high- and low-redshift measurements, namely, the Hubble parameter tension [22-24], as well as the $\sigma_{8}$-tension in the large scale structure formation data $[25,26]$.

On the other hand, the authors of Ref. [27] use observational data to analyze a generic type of cosmological interaction and find that its sign changes during the evolution of the universe. Later, motivated by Ref. [27], the authors of Ref. [28] find a sign change in the cosmological interaction which is described by a running coupling in the cosmic interaction between dark energy and dark matter. Reference [29] proposes a model consistent with thermodynamics and observational constraints, where interaction is proportional to the difference between the energy densities of dark components. In the context of this model, there is a natural change in the interaction sign which coincides with the time when dark energy starts to dominate over dark matter during the evolution. This change in sign is explored further in Refs. [30] and [31]. In the former, the authors explore an holographic dark energy scenario, and interactions with sign change are found to be compatible with the generalized second law of thermodynamics; in the latter, the authors analyze a parameterization of the cosmological interaction that changes sign as the scale factor evolves.

In Ref. [32] a dynamical system analysis was performed for a type of cosmological interaction proportional to the deceleration parameter with the dark energy component modeled by a scalar field, a sign change was naturally induced in the interaction term when the sign of the deceleration parameter changes in the transition from a decelerated universe to 
an accelerated one. Finally the authors find that some scaling attractors could alleviate the cosmological coincidence problem. Other examples of cosmological interaction proportional to the deceleration parameter are found in the literature [33-40].

In the present paper we consider an interacting two-fluid model in which the two dark components are described as perfect fluids with constant barotropic indices and coupled with a nonlinear sign-changeable interaction, depending quadratically on the deceleration parameter. The outline of the paper is as follows. In Sect. 2, we propose an interacting two-fluid model and assume a phenomenological cosmological nonlinear sign-changeable interaction between their components. In Sect. 3 we solve exactly the source equation following the procedure outlined in Ref. [41] for linear and nonlinear interactions and obtain the total energy densities of the dark sector and their dark components as well as the effective equation of state. In Sect. 4 we obtain the late time behavior of models of cosmological interest by using observational data from type Ia supernovae along with measurements of the Hubble expansion rate. Finally, the concluding remarks of this work are presented in Sect. 5 for a large set of factorizable nonlinear sign-changeable interactions.

\section{Interacting dark sector with sign-changeable interaction}

Cosmological interaction was mainly introduced to address the late accelerated expansion of the universe, as well as the cosmic coincidence problem of the standard cosmological scenario ( $\Lambda \mathrm{CDM})$ [7-9]. In this section we investigate interacting scenarios in which the dark components are coupled through sign-changeable interactions $Q$, which are proportional to a quadratic polynomial on the deceleration parameter $q$. This combination includes most of the sign-changeable interactions investigated in the literature [32-39].

We consider an interacting dark sector for a spatially flat FLRW universe with the line element

$\mathrm{d} s^{2}=\mathrm{d} t^{2}-a^{2}(t)\left(\mathrm{d} X^{2}+\mathrm{d} Y^{2}+\mathrm{d} Z^{2}\right)$,

where $t$ is the cosmic time, $a(t)$ is the scale factor, $H=\dot{a} / a$ is the Hubble expansion rate and a dot denotes a derivative with respect to the cosmic time. Dark matter and dark energy have energy densities $\rho_{m}$ and $\rho_{x}$, respectively, so that

$\rho=\rho_{m}+\rho_{x}$,

$\rho^{\prime}=-\gamma_{m} \rho_{m}-\gamma_{x} \rho_{x}$

where $\rho$ is the total energy density and (3) is the conservation equation, the comma indicates derivative with respect to the (time) variable $\eta=\ln \left(a^{3} / a_{0}^{3}\right)$ and $a_{0}$ is some value of reference for the scale factor. For the two components we assume equations of state $p_{m}=\left(\gamma_{m}-1\right) \rho_{m}$ and $p_{x}=\left(\gamma_{x}-1\right) \rho_{x}$, where both barotropic indices $\gamma_{m}$ and $\gamma_{x}$ will be considered constants and satisfying the inequality $\gamma_{x}<\gamma_{m}$ throughout this paper. Solving the algebraic system of Eqs. (2) and (3) for $\rho_{m}$ and $\rho_{x}$ in terms of $\rho$ and $\rho^{\prime}$, we have

$\rho_{m}=-\frac{\gamma_{x} \rho+\rho^{\prime}}{\Delta}, \quad \rho_{x}=\frac{\gamma_{m} \rho+\rho^{\prime}}{\Delta}$,

where $\Delta=\gamma_{m}-\gamma_{x}$ is the determinant of the algebraic system of Eqs. (2) and (3). By using the Friedmann equation we can write the deceleration parameter as a function of the energy density and its $\eta$-derivative,

$q=-\left(1+\frac{3}{2} \frac{\rho^{\prime}}{\rho}\right)$

We begin by introducing a factorizable sign-changeable interaction depending quadratically on the deceleration parameter in the form $Q=\rho\left[q_{1}+q_{2} q+q_{3} q^{2}\right]$, where $q_{1}, q_{2}, q_{3}$ are constants. Combining (5) with the proposed interaction $Q$, we find

$Q\left(\rho, \rho^{\prime}\right)=\rho\left(s_{1}+s_{2} \frac{\rho^{\prime}}{\rho}+s_{3}\left[\frac{\rho^{\prime}}{\rho}\right]^{2}\right)$,

where the constants $s_{i}$ are redefinitions of $q_{i}$. In the first column of Table 1, we show five sign-changeable interactions investigated in the literature that result to depend quadratically on the deceleration parameter. At first sight the interactions in the first column of Table 1 appear to be proportional to the deceleration parameter only; however, when one combines (4) and (5), we find that the functions $\rho, \rho^{\prime}, \rho_{m}, \rho_{x}$ and $q$ are related among them. Thus, in the second column of Table 1 we have written these interactions in terms of $\rho$ and $\rho^{\prime}$, following the form given in (6). In Table 1 we show a set of interactions $Q_{1}-Q_{4}$ investigated in the literature which are included in the general interaction $Q$. In what follows, we will examine the cosmological consequences of the interaction $Q$ in an accelerated scenario for the universe by using the exact dark sector energy density.

From comparing the factorizable interaction (6) with the set of interactions $Q_{1}-Q_{4}$ and $Q$, we find that the coefficients $s_{1}, s_{2}$ and $s_{3}$ satisfy the following constraint:

$s_{3}-\frac{3}{2} s_{2}=-\frac{9}{4} s_{1}$,

meaning that the interactions in Table 1 include no more than two interaction parameters.

Cosmological scenarios driven by other interactions depending on $q$, such as the interactions presented in Refs. [39,42], include higher order derivatives of $\rho$ such as $\rho^{\prime \prime} \rho^{\prime} / \rho$, which is equivalent to considering an interaction proportional to a quadratic polynomial in $q$ with additional terms proportional to $q q^{\prime}$ and they lead to a source equation which becomes nonintegrable by the procedure used in Ref. [41]. 
Table 1 In the left column we present five sign-changeable interactions investigated in the literature that involve the deceleration parameter $q$, while in the right column we write these interactions in terms of $\rho$ and $\rho^{\prime}$ according to (6). Here, $\alpha_{i}, \alpha$ and $\beta$ are interaction parameters

\begin{tabular}{ll}
\hline Interactions & $Q\left(\rho, \rho^{\prime}\right)$ \\
\hline$\alpha_{1} \rho q$ & $Q_{1}=-\alpha_{1} \rho\left(1+\frac{3}{2} \frac{\rho^{\prime}}{\rho}\right)$ \\
$\alpha_{2} \rho^{\prime} q$ & $Q_{2}=-\alpha_{2} \rho\left(\frac{\rho^{\prime}}{\rho}+\frac{3}{2}\left[\frac{\rho^{\prime}}{\rho}\right]^{2}\right)$ \\
$\alpha_{3} \rho_{m} q$ & $Q_{3}=\frac{\alpha_{3}}{\Delta} \rho\left(\gamma_{x}+\left(1+\frac{3}{2} \gamma_{x}\right) \frac{\rho^{\prime}}{\rho}+\frac{3}{2}\left[\frac{\rho^{\prime}}{\rho}\right]^{2}\right)$ \\
$\alpha_{4} \rho_{x} q$ & $Q_{4}=-\frac{\alpha_{4}}{\Delta} \rho\left(\gamma_{m}+\left(1+\frac{3}{2} \gamma_{m}\right) \frac{\rho^{\prime}}{\rho}+\frac{3}{2}\left[\frac{\rho^{\prime}}{\rho}\right]^{2}\right)$ \\
$\left(\alpha \rho+\beta \rho^{\prime}\right) q$ & $Q=-\rho\left(\alpha+\left(\beta+\frac{3}{2} \alpha\right) \frac{\rho^{\prime}}{\rho}+\frac{3}{2} \beta\left[\frac{\rho^{\prime}}{\rho}\right]^{2}\right)$ \\
\hline
\end{tabular}

\section{Solvable sign-changeable interaction scenario}

We split the conservation equation (3) into two coupled first order differential equations,

$$
\begin{aligned}
\rho_{m}^{\prime}+\gamma_{m} \rho_{m} & =-Q, \\
\rho_{x}^{\prime}+\gamma_{x} \rho_{x} & =Q,
\end{aligned}
$$

where we have introduced the phenomenological interaction $Q$ that generates the exchange of energy between dark matter and dark energy components. By differentiating any of Eq. (4) and combining with (8) or (9), we obtain the second order differential equation that determines the energy density for a given interaction $Q$,

$\rho^{\prime \prime}+\left(\gamma_{m}+\gamma_{x}\right) \rho^{\prime}+\gamma_{m} \gamma_{x} \rho=\Delta Q$.

This "source equation" was solved for linear and nonlinear interactions where the former includes a linear combination of the dark matter and dark energy densities, the total energy density and their first derivative; see Ref. [41]. The nonlinear case consists of the above linear combination and additionally significant nonlinear terms having the form of a rational function of the dark matter and dark energy densities. By inserting the sign-changeable interaction (6) into Eq. (10) and making the change of variable $X=\rho^{1+b_{2}}$, valid for $b_{2} \neq-1$, it transforms into the equation of a forced dissipative $\left(b_{1}>0\right)$ or anti-dissipative $\left(b_{1}<0\right)$ linear oscillator,

$X^{\prime \prime}+b_{1} X^{\prime}+b_{3}\left(1+b_{2}\right) X=0$,

where $b_{1}=\gamma_{m}+\gamma_{x}-s_{2} \Delta, b_{2}=-s_{3} \Delta, b_{3}=\gamma_{m} \gamma_{x}-s_{1} \Delta$ are the new parameters of the nonlinear interacting model. Then, the general solution of Eq. (10) is obtained from a "nonlinear superposition" of the two basis solutions of the source equation (11):

$\rho(a)=\left(c_{1} a^{3 \lambda_{1}}+c_{2} a^{3 \lambda_{2}}\right)^{1 /\left(1+b_{2}\right)}$, where

$\lambda_{1,2}=\frac{-b_{1} \pm \sqrt{b_{1}^{2}-4 b_{3}\left(1+b_{2}\right)}}{2}$

are the characteristic roots of the linear differential equation (11). From now on, the $c_{i}$ with $i=1,2, \ldots, n$ will denote arbitrary integration constants. By inserting the energy density (12) into (4), we find the dark matter and dark energy densities to be given by

$$
\begin{gathered}
\rho_{m}=-\frac{\left[\lambda_{2}+\gamma_{x}\left(1+b_{2}\right)\right] \rho+c_{1}\left(\lambda_{1}-\lambda_{2}\right) \frac{a^{3 \lambda_{1}}}{\rho^{b_{2}}}}{\left(1+b_{2}\right) \Delta}, \\
\rho_{x}=\frac{\left[\lambda_{2}+\gamma_{m}\left(1+b_{2}\right)\right] \rho+c_{1}\left(\lambda_{1}-\lambda_{2}\right) \frac{a^{3 \lambda_{1}}}{\rho^{b_{2}}}}{\left(1+b_{2}\right) \Delta} .
\end{gathered}
$$

On obtaining $\rho$, we also have solved the interacting dark sector model for the individual dark matter and dark energy components. However, we can also interpret this interacting dark sector model as a unified cosmological model with effective energy density $\rho$ and effective equation of state $p=-\rho-\rho^{\prime}$ given by

$p=-\left(1+\frac{\lambda_{2}}{1+b_{2}}\right) \rho-\frac{c_{1}\left(\lambda_{1}-\lambda_{2}\right) a^{3 \lambda_{1}}}{\left(1+b_{2}\right) \rho^{b_{2}}}$.

The equation of state (16) includes various of the modified Chaplygin gas models investigated in Refs. [43-47]. Also, nonlinear interactions that give rise to the equation of state (16) allow for the possibility of producing finite-time future singularities [48-50]. In fact, for $\operatorname{sgn}\left(c_{1}\right) \neq \operatorname{sgn}\left(c_{2}\right)$ the parentheses in the energy density (12) vanish for some finite value $a_{s}$ of the scale factor, meaning that both the energy density (12) and the pressure (16) diverge whenever $b_{2}<-1$. Hence, in the case that $\rho_{s}=\rho\left(a_{s}\right)$ diverges at $a_{s}=a\left(t_{s}\right)$ with $t_{s}<\infty$, a finite-time future singularity could occur at the cosmic time $t_{s}$.

By evaluating the energy densities (14) and (15) at the present time, such that $a_{0}=1$, and denoting the density parameters as $\Omega_{m 0}=\rho_{m 0} / 3 H_{0}^{2}$ and $\Omega_{x 0}=\rho_{x 0} / 3 H_{0}^{2}$, where $H_{0}=100 h[\mathrm{~km} / \mathrm{s} / \mathrm{Mpc}]$ is the Hubble parameter, we get

$$
\begin{aligned}
\rho(a)= & 3 H_{0}^{2}\left[\frac{\lambda_{2}+\gamma_{0}\left(1+b_{2}\right)}{\lambda_{2}-\lambda_{1}}\right]^{1 /\left(1+b_{2}\right)} \\
& \times\left(a^{3 \lambda_{1}}-\frac{\lambda_{1}+\gamma_{0}\left(1+b_{2}\right)}{\lambda_{2}+\gamma_{0}\left(1+b_{2}\right)} a^{3 \lambda_{2}}\right)^{1 /\left(1+b_{2}\right)},
\end{aligned}
$$

where $\gamma_{0}=\gamma_{m}-\Omega_{x 0} \Delta$.

From the energy densities (4) we obtain the cosmic coincidence parameter $r(a)=\rho_{m} / \rho_{x}$, which becomes positive for the effective barotropic index $\gamma=-\rho^{\prime} / \rho$ ranging between $\gamma_{x}$ and $\gamma_{m}$. Furthermore, evaluating the coincidence parameter in the limit $a \rightarrow \infty$, from Eqs. (14) and (15), we get a finite constant value $r_{\infty}$,

$r_{\infty}=-\frac{\lambda_{1}+\gamma_{x}\left(1+b_{2}\right)}{\lambda_{1}+\gamma_{m}\left(1+b_{2}\right)}$, 
showing that the quadratic sign-changeable interactions generate dark sector models that are possible candidates for alleviating the coincidence problem. On the other hand, the deceleration parameter (5) has a finite constant value $q_{\infty}$ in the limit $a \rightarrow \infty$ given by

$q_{\infty}=-1-\frac{3 \lambda_{1}}{2\left(1+b_{2}\right)}$

hence, the models with parameters satisfying the condition $3 \lambda_{1} / 2\left(1+b_{2}\right)>-1$ produce a final accelerated stage.

Three particular cases arise when each one of the physical constant parameters $b_{1}, b_{2}$ or $b_{3}$ vanishes. For $b_{1}=0$ the parameter $s_{2}=\left(\gamma_{m}+\gamma_{x}\right) / \Delta$ and the linear term proportional to $\rho^{\prime}$ in the interaction in (6) cancels with the dissipative/antidissipative term in the source equation (10). In this case, the effective dark fluid is described by a linear oscillator with characteristic roots $\lambda_{1}=-\lambda_{2}=\sqrt{-b_{3}\left(1+b_{2}\right)}$. For $b_{2}=0$ the parameter $s_{3}=0$ and the characteristic roots are $\lambda_{1,2}=\left[-b_{1} \pm \sqrt{b_{1}^{2}-4 b_{3}}\right] / 2$, thus we have an energy density that becomes a linear superposition of two different powers of the scale factor. So, the absence of the term proportional to $\rho^{\prime 2} / \rho$ in the source equation (10) has removed the nonlinear superposition effects in the energy density (12). For $b_{3}=0$, we have $s_{1}=\gamma_{m} \gamma_{x} / \Delta, \lambda_{1}=0$ and $\lambda_{2}=b_{1}$, so that the effective energy density and pressure describe a modified Chaplygin gas or a modified anti-Chaplygin gas according to $c_{1} / c_{2}>0$ with $c_{1}>0$ and $c_{2}>0$ or $c_{1} / c_{2}<0$, respectively. In the former case, for a positive $b_{1}$, the universe has a final de Sitter stage with an effective cosmological constant given by the limit $\Lambda \rightarrow \Lambda_{e}=c_{1}^{1 /\left(1+b_{2}\right)}$, and the model reduces to the modified Chaplygin gas of Ref. [51].

Two different branches of solutions are obtained when the parameters $b_{1}=0$ and $b_{3}=0$ or $b_{2}=-1$. In the former case the linear source equation (11) reduces to $X^{\prime \prime}=0$, its general solution is $X=c_{3}+c_{4} \eta$, the barotropic index becomes $\gamma=-c_{4} \rho^{-b_{2}-1} /\left(b_{2}+1\right)$ and the equation of state (16) reduces to

$p=-\rho-\frac{c_{4}}{b_{2}+1} \rho^{-b_{2}}$

This equation can be interpreted as a possible generalization of the polytropic equation of state $p=K \rho^{\gamma p}$, where $K$ is a constant and $\gamma_{p}=-b_{2}$ is the polytropic index. In the latter case, $b_{2}=-1$, the solution of Eq. (11) leads to two different barotropic indices, depending on the values of $b_{1}$; they read

$\gamma=\frac{b_{3}}{b_{1}}-c_{5} a^{-3 b_{1}}, \quad b_{1} \neq 0$,

$\gamma=c_{6}-b_{3} \log \left(a^{3}\right), \quad b_{1}=0$.

These two scenarios could lead to a positive or negative barotropic index regardless of the sign of the constants $b_{3}$, $c_{5}$ and $c_{6}$. In the literature there are other examples of loga- rithmic equations of state as that seen in Eq. (22); see, e.g., $[52,53]$.

The initial sign-changeable interaction (6) can be enlarged by including a term proportional to the first $\eta$-derivative of the deceleration parameter $Q_{q^{\prime}} \propto \rho q^{\prime}$. This generates an additional term in (6), so that it takes the new form $Q_{q^{\prime}}=$ $Q+s_{4} \rho^{\prime \prime}$, with $s_{4}$ an arbitrary constant. However, it is easy to see that the new term $s_{4} \rho^{\prime \prime}$ modifies the coefficient of $\rho^{\prime \prime}$ in the source equation $(10)$, so that $\rho^{\prime \prime} \rightarrow\left(1-s_{4} \Delta\right) \rho^{\prime \prime}$; then we solve for $\rho^{\prime \prime}=\left[Q \Delta-\gamma_{m} \gamma_{x} \rho-\left(\gamma_{m}+\gamma_{x}\right) \rho^{\prime}\right] / s$, where $s=1-s_{4} \Delta$ and $Q_{q^{\prime}}$ becomes

$Q_{q^{\prime}}=\frac{1}{s}\left\{Q-s_{4} \rho\left[\gamma_{m} \gamma_{x}+\left(\gamma_{m}+\gamma_{x}\right) \frac{\rho^{\prime}}{\rho}\right]\right\}$.

The effect of the new term redefines the coefficients in the sign-changeable interaction $Q$, thus we obtain a new $Q_{q^{\prime}}$ that has the same form of the sign-changeable interaction (6). A particular case of this interaction was studied in Ref. [39].

With the above analysis we have an overview of the various effects produced by the nonlinear sign-changeable interaction (6) on the dark sector components; these results were obtained from the general solution of the source equation (11).

\section{Observational analysis}

In this section the observational analysis of the signchangeable interactions $Q_{1}-Q_{4}$ and $Q$ in Table 1 is performed. We consider as free parameters $\alpha_{i}, \alpha$ and $\beta$, describing the cosmological interaction besides $h, \Omega_{m 0}$ and $\gamma_{x}$. Also, for the sake of simplicity, we consider $\gamma_{m}=1$ in all the observational analyses. We use type Ia supernovae ( $\mathrm{SNe}$ ) data from the joint light-curve analysis (JLA) sample [54] with $N=740$ supernovae over the redshift range $0.01<z<1.2$. We obtain the apparent magnitude of a supernovae in the Bband to find

$m(z, \mathbf{p})=\mu(z, \mathbf{p})+M_{B}-\alpha_{*} X_{1}+\beta_{*} \mathcal{C}$,

where $X_{1}$ is the time stretching of the light curve, $\mathcal{C}$ is the supernova color at its maximum brightness, $M_{B}, \alpha_{*}, \beta_{*}$ are nuisance parameters (see Ref. [19] for more details), $\mathbf{p}$ represents the model's parameters and the distance modulus is given by

$\mu(z, \mathbf{p})=5 \log _{10}\left[\frac{d_{L}(z, \mathbf{p})}{10 \mathrm{pc}}\right]$,

for the luminosity distance defined as $d_{L}(z, \mathbf{p})=(1+$ z) $r(z, \mathbf{p})[55]$ and

$r(z, \mathbf{p})=\int_{0}^{z} \frac{c \mathrm{~d} z}{H(z, \mathbf{p})}$. 
We assumed a multivariate Gaussian likelihood in the Monte Carlo analyses for the JLA sample,

$\mathcal{L}_{\mathrm{JLA}}(\mathbf{p})=\exp \left(-\chi_{*}^{2}(\mathbf{p}) / 2\right)$

where

$\chi_{*}^{2}(\mathbf{p})=\left(m_{B}-m(z, \mathbf{p})\right)^{T} C_{*}^{-1}\left(m_{B}-m(z, \mathbf{p})\right)$,

and $C_{*}$ is the covariance matrix of the $m_{B}$ measurements, estimated accounting for statistical and systematic uncertainties (see Ref. [19] for more details).

Additionally, we consider 51 measurements of the Hubble expansion rate (see Table 1 of Ref. [56]) where the data come from the differential age method for passively evolving galaxies or indirectly through BAO measurements. The $\chi^{2}$ function for the Hubble expansion rate is defined by

$\chi_{H}^{2}=\sum_{i}\left(\frac{H_{\mathrm{obs}, i}-H_{\mathrm{th}}\left(z_{i} ; \mathbf{p}\right)}{\sigma_{H}\left(z_{i}\right)}\right)^{2}$,

where the subscripts obs and th mean observational values and theoretical predictions, respectively; $\sigma_{H}$ denotes the measurement's error and the likelihood is defined in (27).

From now on we use $H(z)=\sqrt{\rho / 3}$, where $\rho$ is given by (17) rewritten in terms of the cosmological redshift $z$. The constants $\lambda_{1}, \lambda_{2}, b_{2}$ and $k$ in Eq. (17) are rewritten in terms of the model's parameters $\mathbf{p}=\left(h,\left\{\alpha_{i}, \alpha, \beta\right\}, \Omega_{m 0}, \gamma_{x}\right)$.

In order to find the best fit parameters, we use the Python interface for the MULTINEST algorithm [57-59], where we assume the following uniform priors: $\alpha_{i} \in[-1,1], \beta \in$ $[-1,1], \Omega_{m 0} \in[0,1]$ and $\gamma_{x} \in[-1,1]$. In order to break the degeneracy in the $h$ parameter we consider the joint analyses JLA $+H_{0}$ and JLA $+H_{0}+H(z)$, where $H_{0}$ is the local measurement for the Hubble parameter [60].

The best fit parameters and the $1 \sigma$ error are shown in Tables 2, 3, 4 and 5 where we have considered interactions defined in Table 1. In order to focus on the interaction parameters we firstly use $\gamma_{x}=0$ as prior in Tables 2 and 3, where we also include the fit of the $\Lambda \mathrm{CDM}$ model. In Tables 4 and 5 we enlarge the parameter space by including $\gamma_{x}$ as a free parameter. In these tables we include the fit of the $\omega \mathrm{CDM}$ model as comparison instead of $\Lambda \mathrm{CDM}$, given that the former considers the state parameter of the dark energy $\omega$ as a free parameter. In Tables 2 and 4 we show the analysis of the interactions in Table 1 considering JLA $+H_{0}$ and in Tables 3 and 5 we expand the data set to include $H(z)$.

We notice in Tables 2 and 4 that the values of the interaction parameters, $\alpha_{i}, \alpha$ and $\beta$, are all consistent with zero (within the $1 \sigma$ region). Nevertheless, only scenarios $Q_{4}$ and $Q$ are consistent with no interaction in Table 3 and only scenario $Q$ in Table 5. This means that it is necessary to add the $H(z)$ data in order to get a defined sign of the interaction in most of the scenarios. On the other hand, only interaction $Q_{1}$
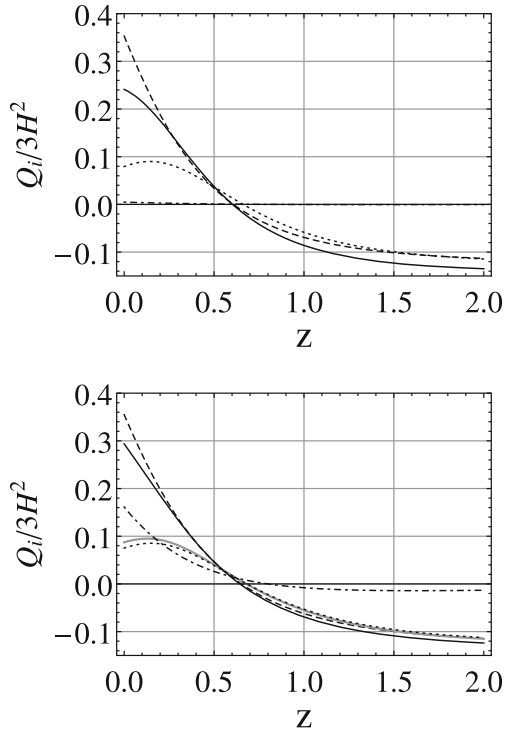

Fig. 1 Evolution of a dimensionless interaction term. The top panel corresponds to $\gamma_{x}=0$ and the bottom panel to a fitted $\gamma_{x}$, both figures consider the analysis JLA $+H_{0}+\mathrm{H}(\mathrm{z})$. The black lines are for interacting models, dashed, dotted, dot-dashed and solid lines represent interactions $Q_{1}, Q_{3}, Q_{4}$ and $Q$, respectively. On the top panel $Q_{2}$ is indistinguishable from $Q_{3}$, on the other panel $Q_{2}$ corresponds to the gray line

and $Q$ maintain the same sign of interaction parameters for all the results in Tables 2, 3, 4 and 5 .

The results in Tables 4 and 5 show a positive $\gamma_{x}$ in any case, nevertheless this value is consistent with zero in some cases. In comparing Table 2 with 3 and Table 4 with 5 for interacting scenarios, we can see that the values of the parameters $h$ and $\Omega_{m 0}$ become smaller including the $H(z)$ data. This is natural given that we are using a Gaussian prior to set the value of $h$ in obtaining the results of Tables 2 and 4, that is, the SNe data do not restrict the $h$ parameter by themselves. In including the $H(z)$ data in Tables 3 and 5 the $h$ posterior changes, because this set of data is closely related to the $h$ parameter.

In comparing Table 2 with 4 and 3 with 5 we notice that, on including the $\gamma_{x}$ parameter, the value of $h$ is approximately the same; nevertheless, the $\Omega_{m 0}$ parameter always diminishes. On the other hand, in comparing the full analyses in Tables 3 and 5, we observe that the sign of the interaction is maintained in each case. We additionally notice that the fit for interactions $Q_{2}$ and $Q_{3}$ are very similar in Tables 2 and 3 , and this is consistent with the fact that the two correspond to the same interaction up to a sign in the case $\gamma_{x}=0$ and $\gamma_{m}=1$, as we can observe from Table 1 .

In Fig. 1 we show the evolution of the interaction term and we observe that all the studied interactions, $Q_{1}-Q_{4}$ and $Q$, correspond to a positive interacting term today, i.e., we have energy transfer from dark matter to dark energy today. Besides, the change of sign of the interaction took place in 
Table 2 Best fit parameters and $1 \sigma$ error for the analysis using $\mathrm{JLA}+\mathrm{H}_{0}$. We have considered $\gamma_{x}=0$ and as a comparison we show the fit for $\Lambda \mathrm{CDM}$
Table 3 Best fit parameters and $1 \sigma$ error for the analysis using $\mathrm{JLA}+H_{0}+H(z)$. We have considered $\gamma_{x}=0$ and as a comparison we show the fit for $\Lambda \mathrm{CDM}$

\begin{tabular}{llllll}
\hline Scenario & $h$ & $\alpha_{i}$ or $\alpha$ & $\beta$ & $\Omega_{m 0}$ & $\chi_{\min }^{2}$ \\
\hline$\Lambda$ CDM & $0.732 \pm 0.016$ & - & - & $0.298 \pm 0.033$ & 682.901 \\
$Q_{1}$ & $0.733 \pm 0.016$ & $-0.05_{-0.49}^{+0.54}$ & - & $0.286_{-0.092}^{+0.13}$ & 683.033 \\
$Q_{2}$ & $0.733 \pm 0.016$ & $-0.13_{-0.78}^{+0.37}$ & - & $0.310_{-0.050}^{+0.057}$ & 682.769 \\
$Q_{3}$ & $0.732 \pm 0.016$ & $0.13_{-0.37}^{+0.79}$ & - & $0.310_{-0.051}^{+0.058}$ & 682.766 \\
$Q_{4}$ & $0.732 \pm 0.016$ & $0.11_{-0.63}^{+0.37}$ & - & $0.308_{-0.057}^{+0.083}$ & 682.802 \\
$Q$ & $0.733 \pm 0.016$ & $-0.01_{-0.44}^{+0.57}$ & $0.05_{-0.64}^{+0.49}$ & $0.293_{-0.084}^{+0.13}$ & 682.993 \\
\hline
\end{tabular}

\begin{tabular}{llllll}
\hline Scenario & $h$ & $\alpha_{i}$ or $\alpha$ & $\beta$ & $\Omega_{m 0}$ & $\chi_{\min }^{2}$ \\
\hline$\Lambda$ CDM & $0.7106 \pm 0.0089$ & - & - & $0.256 \pm 0.013$ & 711.966 \\
$Q_{1}$ & $0.707 \pm 0.010$ & $-0.44_{-0.36}^{+0.22}$ & - & $0.132_{-0.11}^{+0.054}$ & 711.259 \\
$Q_{2}$ & $0.7076 \pm 0.0098$ & $0.54_{-0.15}^{+0.42}$ & - & $0.218_{-0.033}^{+0.023}$ & 709.588 \\
$Q_{3}$ & $0.707 \pm 0.010$ & $-0.54_{-0.43}^{+0.14}$ & - & $0.219_{-0.034}^{+0.022}$ & 709.508 \\
$Q_{4}$ & $0.709_{-0.011}^{+0.010}$ & $-0.01 \pm 0.31$ & - & $0.249_{-0.062}^{+0.084}$ & 712.582 \\
$Q$ & $0.7063_{-0.011}^{+0.0096}$ & $-0.25 \pm 0.34$ & $0.42_{-0.23}^{+0.53}$ & $0.159_{-0.095}^{+0.082}$ & 709.786 \\
\hline
\end{tabular}

Table 4 Best fit parameters and $1 \sigma$ error for the analysis using $\mathrm{JLA}+H_{0}$. As a comparison we show the fit for $\omega \mathrm{CDM}$

\begin{tabular}{lllllll}
\hline Scenario & $h$ & $\alpha_{i}$ or $\alpha$ & $\beta$ & $\Omega_{m 0}$ & $\gamma_{x}$ & $\chi_{\min }^{2}$ \\
\hline$\omega \mathrm{CDM}$ & $0.732 \pm 0.016$ & - & - & $0.220 \pm 0.110$ & $0.13_{-0.12}^{+0.26}$ & 683.017 \\
$Q_{1}$ & $0.733 \pm 0.016$ & $-0.05 \pm 0.46$ & - & $0.238_{-0.21}^{+0.094}$ & $0.07_{-0.13}^{+0.32}$ & 683.495 \\
$Q_{2}$ & $0.732 \pm 0.016$ & $0.01 \pm 0.53$ & - & $0.210 \pm 0.110$ & $0.14_{-0.12}^{+0.24}$ & 683.409 \\
$Q_{3}$ & $0.732 \pm 0.016$ & $0.12_{-0.39}^{+0.81}$ & - & $0.24_{-0.16}^{+0.11}$ & $0.11_{-0.12}^{+0.30}$ & 682.885 \\
$Q_{4}$ & $0.732 \pm 0.016$ & $0.02_{-0.57}^{+0.29}$ & - & $0.230_{-0.160}^{+0.130}$ & $0.09_{-0.14}^{+0.28}$ & 684.520 \\
$Q$ & $0.732 \pm 0.016$ & $-0.03 \pm 0.48$ & $0.01_{-0.63}^{+0.54}$ & $0.234_{-0.21}^{+0.094}$ & $0.09_{-0.12}^{+0.30}$ & 683.548 \\
\hline
\end{tabular}

Table 5 Best fit parameters and $1 \sigma$ error for the analysis using $\mathrm{JLA}+H_{0}+H(z)$. As a comparison we show the fit for $\omega \mathrm{CDM}$

\begin{tabular}{lllllll}
\hline Scenario & $h$ & $\alpha_{i}$ or $\alpha$ & $\beta$ & $\Omega_{m 0}$ & $\gamma_{x}$ & $\chi_{\min }^{2}$ \\
\hline$\omega \mathrm{CDM}$ & $0.7042_{-0.011}^{+0.0098}$ & - & - & $0.251 \pm 0.015$ & $0.053_{-0.053}^{+0.059}$ & 710.028 \\
$Q_{1}$ & $0.7031_{-0.011}^{+0.0096}$ & $-0.49_{-0.29}^{+0.19}$ & - & $0.103_{-0.094}^{+0.037}$ & $0.090_{-0.065}^{+0.079}$ & 708.426 \\
$Q_{2}$ & $0.7044_{-0.011}^{+0.0094}$ & $0.57_{-0.14}^{+0.40}$ & - & $0.207 \pm 0.036$ & $0.039_{-0.064}^{+0.084}$ & 708.559 \\
$Q_{3}$ & $0.7042_{-0.011}^{+0.0098}$ & $-0.55_{-0.43}^{+0.14}$ & - & $0.214_{-0.033}^{+0.025}$ & $0.035_{-0.062}^{+0.080}$ & 708.578 \\
$Q_{4}$ & $0.7047_{-0.011}^{+0.0099}$ & $-0.307_{-0.35}^{+0.088}$ & - & $0.166_{-0.099}^{+0.052}$ & $0.097_{-0.054}^{+0.080}$ & 709.747 \\
$Q$ & $0.7030_{-0.011}^{+0.0093}$ & $-0.37_{-0.39}^{+0.27}$ & $0.22_{-0.34}^{+0.69}$ & $0.124_{-0.11}^{+0.050}$ & $0.077_{-0.065}^{+0.095}$ & 708.344 \\
\hline
\end{tabular}

the past (around $z \sim 0.7$ ), concurrently with the deceleration parameter transition in each case.

Figure 2 shows the effective barotropic index, which as a result appears to be positive today in each case; in Fig. 3 we show the evolution of the energy density parameter for the dark energy and we notice that the dark energy contribution today is always higher for the studied interacting scenarios, compared to the standard scenario $\Lambda \mathrm{CDM}$. This is consistent with energy transfer from dark matter to dark energy. Furthermore, from Figs. 1, 2 and 3 we realize that the interaction $Q_{4}$ (dot-dashed line) corresponds to the sign- changeable scenario closest to the $\Lambda \mathrm{CDM}$ or the $\omega \mathrm{CDM}$ evolution.

In Figs. 4 and 5 we show as examples the contour plot of interaction $Q_{4}$ in Table 1. Figure 4 has the $\gamma_{x}$ parameter set to zero and in Fig. $5 \gamma_{x}$ is allowed to vary. We can see that all the parameters are better constrained in the analysis $\mathrm{JLA}+H_{0}+\mathrm{H}(\mathrm{z})$ compared to the analysis $\mathrm{JLA}+H_{0}$.

Finally, we notice from the results in Tables 2, 3, 4 and 5 that the interacting models $Q_{1}-Q_{4}$ and $Q$ fulfill the condition $b_{2}>-1$; hence according to Eq. (12), we do not expect scenarios with a future singularity. 

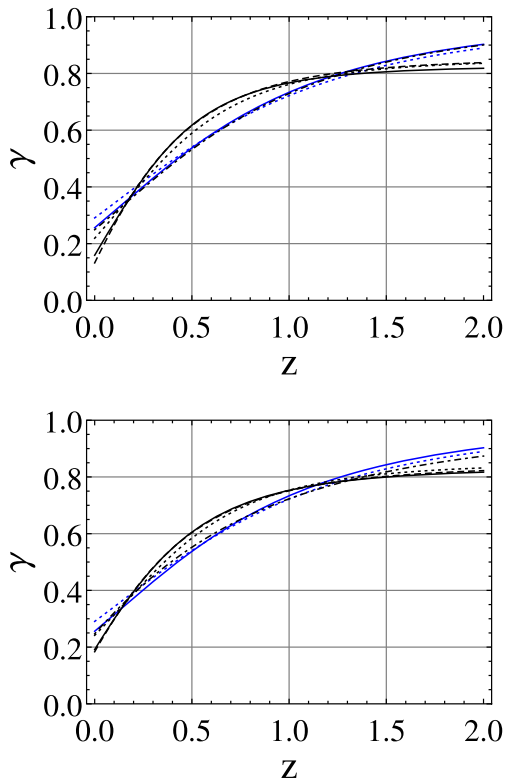

Fig. 2 Evolution of the effective barotropic index. The top panel corresponds to $\gamma_{x}=0$ and the bottom panel to a fitted $\gamma_{x}$, both figures consider the analysis JLA $+H_{0}+\mathrm{H}(\mathrm{z})$. The blue lines represent $\Lambda \mathrm{CDM}$ (solid) and $\omega \mathrm{CDM}$ (dotted). The black lines are for interacting models, dashed, dotted, dot-dashed and solid lines represent interactions $Q_{1}, Q_{3}, Q_{4}$ and $Q$, respectively. In both figures $Q_{2}$ is indistinguishable from $Q_{3}$
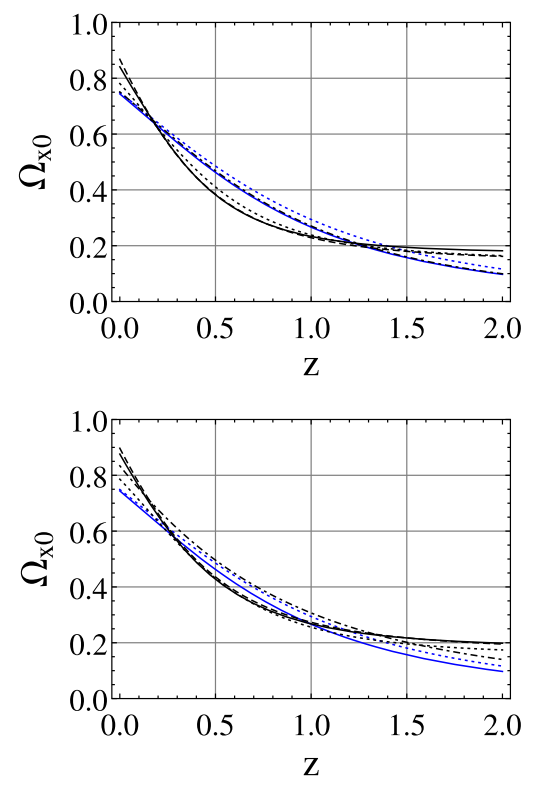

Fig. 3 Evolution of the density parameter for dark energy. The top panel corresponds to $\gamma_{x}=0$ and the bottom panel to a fitted $\gamma_{x}$, both figures consider the analysis JLA $+H_{0}+\mathrm{H}(\mathrm{z})$. The blue lines represent $\Lambda \mathrm{CDM}$ (solid) and $\omega \mathrm{CDM}$ (dotted). The black lines are for interacting models, dashed, dotted, dot-dashed and solid lines represent interactions $Q_{1}, Q_{3}, Q_{4}$ and $Q$, respectively. In both figures $Q_{2}$ is indistinguishable from $Q_{3}$

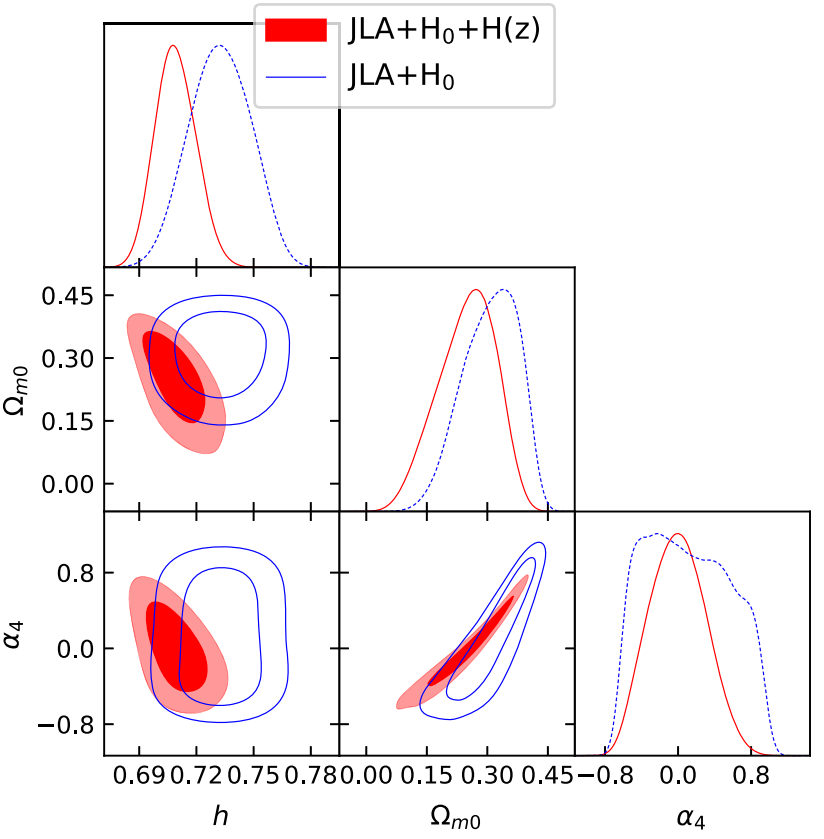

Fig. 4 The contour plot for interaction $Q_{4}$ with the $1 \sigma$ and $2 \sigma$ regions. We considered the analysis JLA $+H_{0}$ (blue) and JLA $+H_{0}+H(z)$ (red)

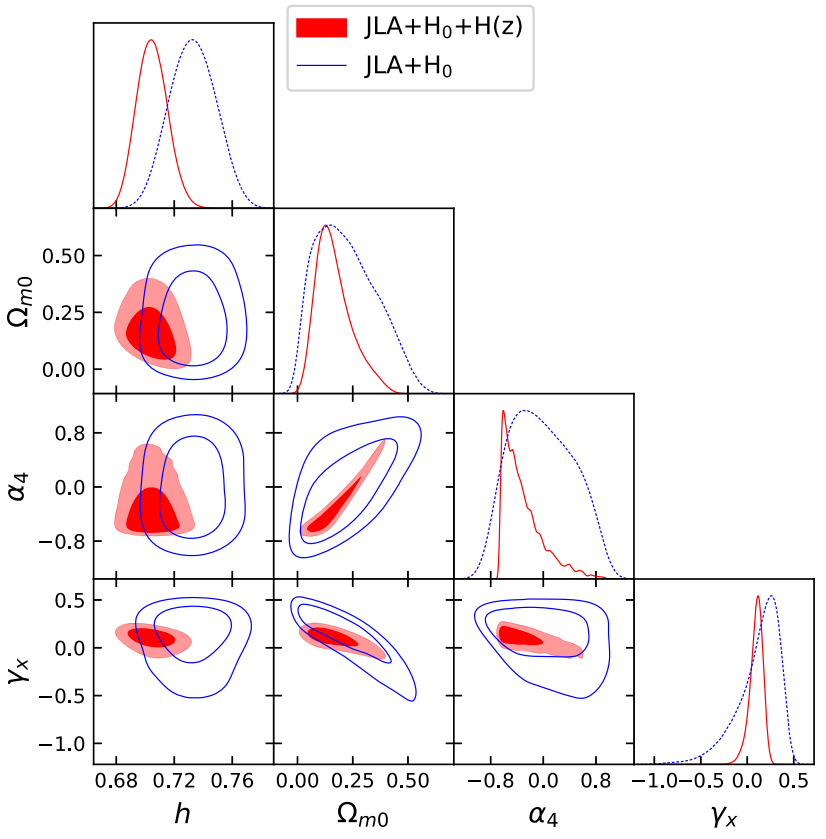

Fig. 5 The contour plot for interaction $Q_{4}$ with the $1 \sigma$ and $2 \sigma$ regions. We considered the analysis JLA $+H_{0}$ (blue) and JLA $+H_{0}+H(z)$ (red) 


\section{Final discussion}

We have presented an interacting dark sector scenario in a spatially flat FLRW and we introduced a factorizable nonlinear sign-changeable interaction between the dark components depending linearly on the energy density and quadratically on the deceleration parameter, $Q=\rho\left[q_{0}+q_{1} q+\right.$ $\left.q_{2} q^{2}\right]$, resulting in cosmological scenarios with a natural sign change related to the accelerated expansion of the universe.

We have studied several linear and nonlinear signchangeable interactions and have written them in terms of $\rho$ and $\rho^{\prime}$ in Table 1 , by using the deceleration parameter of Eq. (6). The interactions $Q_{1}-Q_{4}$ were previously analyzed in a different context in Ref. [32], by using a dynamical system and considering the cosmological evolution of the dark energy component in the form of quintessence and phantom scalar fields. Instead we have considered a general sign-changeable interaction $Q$ that includes $Q_{1}-Q_{4}$ and we assumed an interacting dark sector composed of dark matter and dark energy with constant barotropic indices. We have focused on a larger set of scenarios which can include an inherent change of sign in the interaction term.

We have shown that different types of sign-changeable interactions are linked by recognizing a convenient form of expanding the interaction terms and therefore integration methods described in [41] can be applied. We have solved the corresponding source equation (10) when it is sourced by $Q$ depending on the deceleration parameter and obtained the effective energy density (12), the dark matter and dark energy densities (14) and (15) as well as the effective equation of state (16). In the particular scenario $b_{3}=0$ in Eq. (11), we have found that the effective equations of state describe the Chaplygin gas and its generalizations, so we have an unified model for the interacting dark sector.

We have used SNe type Ia from the JLA compilation [54] along with $H(z)$ [56] data and the Riess' value for $H_{0}$ [60] to constrain the parameters of the five interactions in Table 1, using the effective energy density (12). The best fit parameters for each model are shown in Tables 2, 3, 4 and 5, from which we obtain $b_{2}>-1$ in any case, then from Table 1 , we could construct an interacting dark model free of finitetime future singularities, consistent with the cosmological data used.

According to the observational data analysis, summarized in Fig. 1 for the evolution of the interaction, an energy transfer occurs from dark matter to dark energy today in all scenarios. Also, as expected a transition is observed in the past where the energy transfer changes direction; this occurs concurrently with the acceleration-deceleration transition. The effective barotropic index as a results appears to be of quintessencetype today in any case; see Fig. 2. Given that the energy transfer is from dark matter to dark energy in all the cases, we see in Fig. 3 that the dark energy density is always larger than the $\Lambda \mathrm{CDM}$ model today.

In a forthcoming work we will perform a full analysis including baryons and photons in order to include more available data such as baryonic acoustic oscillations and cosmic microwave background measurements.

Acknowledgements FA was partially supported from Dirección de Investigación Universidad de La Frontera, Project no. DI17-0075. AC acknowledges the support of Dirección de Investigación Universidad del Bío-Bío through Grant no. GI-172309/C. LPC was supported by Universidad de Buenos Aires under the Project no. 20020100100147 and CONICET under the Project PIP 114-201101-00317.

Data Availability Statement This manuscript has no associated data or the data will not be deposited. [Authors' comment: The data used in this paper are publicly available and they can be found in the corresponding references.]

Open Access This article is distributed under the terms of the Creative Commons Attribution 4.0 International License (http://creativecomm ons.org/licenses/by/4.0/), which permits unrestricted use, distribution, and reproduction in any medium, provided you give appropriate credit to the original author(s) and the source, provide a link to the Creative Commons license, and indicate if changes were made. Funded by SCOAP ${ }^{3}$.

\section{References}

1. A.G. Riess et al., Supernova Search Team Collaboration. Astron. J. 116, 1009 (1998)

2. S. Perlmutter et al., Supernova Cosmology Project Collaboration. Astrophys. J. 517, 565 (1999)

3. E. Komatsu et al., [WMAP Collaboration]. Astrophys. J. Suppl. 192, 18 (2011)

4. P.A.R. Ade et al., [Planck Collaboration]. Astron. Astrophys. 594, A13 (2016)

5. D.M. Scolnic et al., Astrophys. J. 859(2), 101 (2018)

6. K. Bamba, S. Capozziello, S. Nojiri, S.D. Odintsov, Astrophys. Space Sci. 342, 155 (2012)

7. L.P. Chimento, A.S. Jakubi, D. Pavon, W. Zimdahl, Phys. Rev. D 67, 083513 (2003)

8. D. Pavon, W. Zimdahl, Phys. Lett. B 628, 206 (2005)

9. Z.-K. Guo, Y.-Z. Zhang, Phys. Rev. D 71, 023501 (2005)

10. W. Zimdahl, Int. J. Mod. Phys. D 14, 2319 (2005)

11. J.D. Barrow, T. Clifton, Phys. Rev. D 73, 103520 (2006)

12. W. Zimdahl, Int. J. Mod. Phys. D 17, 651 (2008)

13. R.F. vom Marttens, W.S. Hipolito-Ricaldi, W. Zimdahl, JCAP 1408, 004 (2014)

14. F. Arevalo, P. Cifuentes, S. Lepe, F. Pena, Astrophys. Space Sci. 352, 899 (2014)

15. R.F. vom Marttens, L. Casarini, W.Zimdahl, W.S. Hipolito-Ricaldi, D.F. Mota, Phys. Dark Univ. 15, 114 (2017)

16. R.F. vom Marttens, L. Casarini, W.S. Hipolito-Ricaldi, W.Zimdahl, JCAP 1701(01), 050 (2017)

17. S. Kumar, R.C. Nunes, Phys. Rev. D 94(12), 123511 (2016)

18. S. Kumar, R.C. Nunes, Phys. Rev. D 96(10), 103511 (2017)

19. A. Cid, B. Santos, C. Pigozzo, T. Ferreira, J. Alcaniz, JCAP 1903(03), 030 (2019)

20. F. Arevalo, A. Cid, J. Moya, Eur. Phys. J. C 77(8), 565 (2017)

21. B. Wang, E. Abdalla, F. Atrio-Barandela, D. Pavon, Rep. Prog. Phys. 79(9), 096901 (2016) 
22. E. Di Valentino, A. Melchiorri, O. Mena, Phys. Rev. D 96(4), 043503 (2017)

23. J. Sola, A. Gomez-Valent, J. de Cruz Perez, Phys. Lett. B 774, 317 (2017)

24. W. Yang, S. Pan, E. Di Valentino, R.C. Nunes, S. Vagnozzi, D.F. Mota, JCAP 1809(09), 019 (2018)

25. R. Murgia, arXiv:1612.02282 [astro-ph.CO]

26. A. Gomez-Valent, J. Sola, EPL 120(3), 39001 (2017)

27. R.-G. Cai, Q. Su, Phys. Rev. D 81, 103514 (2010)

28. Y.-H. Li, X. Zhang, Eur. Phys. J. C 71, 1700 (2011)

29. C.-Y. Sun, R.-H. Yue, Phys. Rev. D 85, 043010 (2012)

30. F. Arevalo, P. Cifuentes, F. Pena, Astrophys. Space Sci 361(1), 45 (2016). https://doi.org/10.1007/s10509-015-2634-7

31. J.J. Guo, J.F. Zhang, Y.H. Li, D.Z. He, X. Zhang, Sci. China Phys. Mech. Astron. 61(3), 030011 (2018)

32. H. Wei, Nucl. Phys. B 845, 381-392 (2011)

33. Y.D. Xu, X.H. Zhai, Astrophys. Space Sci. 345, 381-386 (2013)

34. Y.D. Xu, Z.G. Huang, Astrophys. Space Sci. 350, 855-859 (2014)

35. M. Khurshudyan, arXiv:1302.1220 [gr-qc]

36. M. Khurshudyan, A. Khurshudyan, Astrophys. Space Sci. 357(2), 113 (2015)

37. S. Lepe, F. Pena, F. Torres, Phys. Rev. D 91(2), 024023 (2015)

38. H. Wei, Commun. Theor. Phys. 56, 972 (2011)

39. M. Forte, Gen. Relat. Gravit. 46, 1811 (2014)

40. Y.D. Xu, Z.G. Huang, Astrophys. Space Sci. 343, 807 (2013)

41. L.P. Chimento, Phys. Rev. D 81, 043525 (2010)

42. Y.L. Bolotin, A. Kostenko, O.A. Lemets, D.A. Yerokhin, Int. J. Mod. Phys. D 24(03), 1530007 (2014)

43. Z.K. Guo, Y.Z. Zhang, Phys. Lett. B 645, 326 (2007)

44. Z.K. Guo, Y.Z. Zhang, arXiv:astro-ph/0509790
45. G. Sethi, S.K. Singh, P. Kumar, D. Jain, A. Dev, Int. J. Mod. Phys. D 15, 1089 (2006)

46. U. Debnath, Astrophys. Space Sci. 312, 295 (2007)

47. M. Jamil, Int. J. Mod. Phys. D 49, 62-71 (2010)

48. M. Cataldo, L.P. Chimento, M.G. Richarte, Phys. Rev. D 95(6), 063510 (2017)

49. L.P. Chimento, M.G. Richarte, Phys. Rev. D 93(4), 043524 (2016). (erratum: Phys. Rev. D95(6), 069902, 2017)

50. L.P. Chimento, M.G. Richarte, Phys. Rev. D 92(4), 043511 (2015)

51. H.B. Benaoum, arXiv:hep-th/0205140

52. S.D. Odintsov, V.K. Oikonomou, A.V. Timoshkin, E.N. Saridakis, R. Myrzakulov, Annals Phys. 398, 238-253 (2018)

53. W. Yang, S. Pan, A. Paliathanasis, Mon. Not. R. Astron. Soc. 482, 1007 (2019)

54. M. Betoule et al., [SDSS Collaboration]. Astron. Astrophys. 568, A22 (2014)

55. S. Weinberg, Cosmology (Oxford University Press, Oxford, 2008)

56. J. Magana, M.H. Amante, M.A. Garcia-Aspeitia, V. Motta, Mon. Not. R. Astron. Soc. 476(1), 1036 (2018)

57. F. Feroz, M.P. Hobson, Mon. Not. R. Astron. Soc. 384, 449 (2008)

58. F. Feroz, M.P. Hobson, M. Bridges, Mon. Not. R. Astron. Soc. 398, 1601 (2009)

59. F. Feroz, M.P. Hobson, E. Cameron, A.N. Pettitt, arXiv:1306.2144 [astro-ph.IM]

60. A.G. Riess et al., Astrophys. J. 826(1), 56 (2016) 